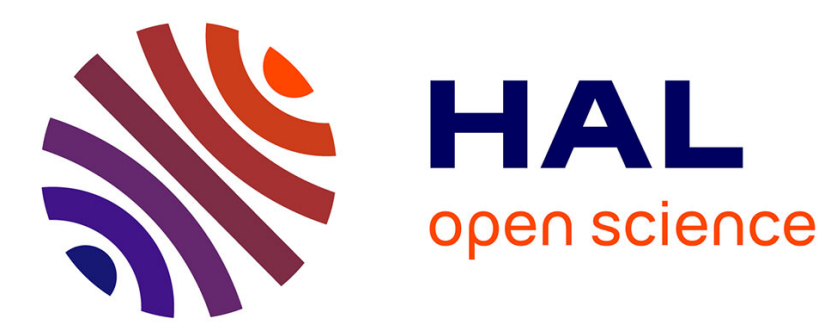

\title{
Électrisation négative des rayons secondaires issus de la transformation des rayons $\mathrm{X}$ \\ Pierre Curie, G. Sagnac
}

\section{To cite this version:}

Pierre Curie, G. Sagnac. Électrisation négative des rayons secondaires issus de la transformation des rayons X. J. Phys. Theor. Appl., 1902, 1 (1), pp.13-21. 10.1051/jphystap:01902001001301 . jpa-00240585

\section{HAL Id: jpa-00240585 https://hal.science/jpa-00240585}

Submitted on 1 Jan 1902

HAL is a multi-disciplinary open access archive for the deposit and dissemination of scientific research documents, whether they are published or not. The documents may come from teaching and research institutions in France or abroad, or from public or private research centers.
L'archive ouverte pluridisciplinaire HAL, est destinée au dépôt et à la diffusion de documents scientifiques de niveau recherche, publiés ou non, émanant des établissements d'enseignement et de recherche français ou étrangers, des laboratoires publics ou privés. 


\section{ÉLEGTRISATION NÉGATIVE DES RAYONS SEGONDAIRES ISSUS DE LA TRANSFORMATION DES RAYONS $\mathrm{X}$;}

Par MM. P. GURIE et G. SAGNAG.

Le faible pouvoir de pénétration des rayons secondaires des métaux lourds fait penser aux rayons cathodiques de Lenard, lesquels peuvent seulement parcourir quelques centimètres à peine dans l'air atmosphérique, où ils sont énergiquement diffusés. Celte analogie conduit à rechercher si les rayons secondaires, très absorbables par l'air, transportent avec eux des charges électriques négatives, puisque tel est le caractère fondamental des rayons cathodiques; la déviation des rayons par le champ magnétique (1) ou par le champ électrique sera une conséquence probable de leur électrisation. Il n'y a pas de contradiction entre cette hypothèse et celles qui ont été développées par l'un de nous, puisque le faisceau émis spontanément par le radium de $\mathrm{M}$. et de $\mathrm{M}^{\mathrm{me}}$ Curie est un mélange de rayons électrisés négativement analogues aux rayons cathodiques, déviables par le champ magnétique et par le champ électrique, et de rayons non déviables analogues aux rayons $X$, sensiblement dépourvus de charges électriques.

Pour préciser d'abord jusqu'à quel point les rayons $\mathrm{X}$ se montrent dépourvus d'électrisation $\left({ }^{2}\right)$, nous employions une encein'e de

(1) P. Curie et G. Sagiac, C. R., t. CXXX, p. $1013 ; 9$ a viril 1900.

(2) Le professeur E. Dorn a annoncé que les rayons secondaires des métaux lourds sont déviés par le champ magnétique, et dans le même sens que les rayons cathodiques (Abhand.d. Naturf. Gesell. zu Halle, Bd. XXII, p. 40-42;1900).

L'un de nous avait antérieurement émis l'opinion que les rayons secondaires très absorbables des métaux lourds peuvent renfermer des rayons analogues à ceux de Lenard et déviables comme eux par l'aimant [G. SAGixac, Recherches sur les transformations des rayons de Röntgen, ch. I, $3^{e}$ paragraphe : Rayons secondaires, rayons $X$ et rayons de Lenard (l'Eclairage électrique du 12 mars 1898)]. 
Faraday en plomb épais de forme cubique, ayant 23 centimètres de côté, reliée à un électromètre à quadrants de Curic. Un large faisceau de rayons $X$ y pénétrait par une ouverture circulaire de 10 centimètres de diamètre, placée à 7 centimètres seulement de la lame focus du tube producteur de rayons $\mathrm{X}$.

L'enceinte de plomb, y compris son ouverture, était complètement enveloppée par une couche continue d'un diélectrique solide (paraffine ou ébonite), recouverte elle-même d'une enveloppe d'aluminium mince en communication avec la terre. L'enveloppe continue de diélectrique solide est nécessaire pour maintenir l'isolement parfait du cylindre qui, sans cette précaution, ne demeurerait pas isolé dans l'air ambiant rendu conducteur de l'électricité par l'action des rayons de Röntgen.

Dans ces conditions, l'électromètre ne se chargeait pas sensiblement. Nous avons pu ainsi conclure qu'en admettant l'hypothèse de rayons $\mathrm{X}$ électrisés, le courant, équivalent à la circulation de l'électricité dans le faisceau large et intense de rayons $\mathrm{X}$ employé, était certainement inférieur à $10^{-12}$ ampère.

Nous avons pu, au contraire, conclure l'électrisation négative des rayons secondaires des métaux lourds. A la pression atmosphérique, les rayons $\mathrm{X}$ et les rayons secondaires communiquent à l'air une conductibilité telle que le métal rayonnant n'est plus isolé; il est alors impossible de recueillir l'électricité des rayons secondaires. Il fallait éviter en même temps que les rayons secondaires des métaux lourds, souvent très peu pénétrants, ne fussent absorbés au voisinage immédiat du métal qui les émet. Nous avons été ainsi amenés à placer les métaux dans l'air raréfié et à opérer à des pressions de plus en plus faibles, jusqu'au vide de Crookes $\left(0^{\mathrm{mm}}, 001\right.$ de mercure), afin de rendre à l'air ses propriétés isolantes, malgré l'action des rayons de Röntgen et des rayons secondaires qui le traversent. Nous avons réduit à 3 ou 4 millimètres seulement la couche d'air raréfié comprise entre le métal rayonnant et les parois métalliques voisines. Dans cette mince couche d'air très raréfié, la force électromotrice entre le métal rayonnant et les parois qui l'entourent produit seulement, sons l'influence des rayons, un courant inférieur, par exemple, à 1/100 du courant dû à l'électricité négative des rayons secondaires d'un métal, tel que le platine, le plomb. Le dispositif est celui-ci :

Une feuille métallique mince $M(f g .1)$ reliée à un électromètre à quadrants et à un quartz piézo-électrique de M. P. Curie, est maintenue 
isolée au milieu et à 3 millimètres seulement des parois d'une boîte métallique plate $\mathrm{ABCD}$, qu'on peut mettre en relation avec la terre. La face inférieure $C D$ de cette boîte est formée, comme la face supérieure $\mathrm{AB}$, d'une plaque épaisse d'un autre métal $\mathrm{N}$, mais percée de

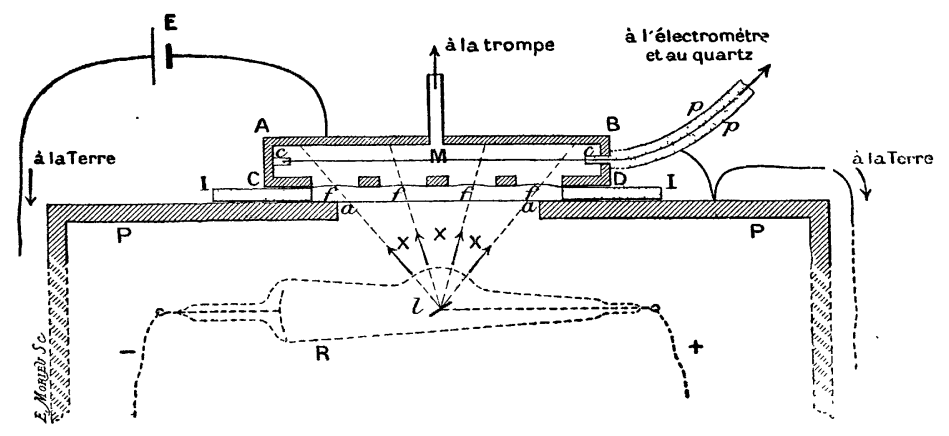

Fig. 1.

fenêtres $f$ que recouvre une mince feuille du métal N. A 6 centimètres au-dessous de la face $\mathrm{AB}$ se trouve la lame focus $l$, source des rayons de Röntgen. Le système producteur de ces rayons (tube focus R, bobine Ruhmkorff et interrupteur électrolytique de Wehnelt) est enfermé dans une grande caisse de plomb épais dont la paroi PP est mise à la terre. Les rayons de Röntgen sortent de la caisse PP par une ouverture circulaire de 10 centimètres de diamètre recouverte seulement d'une mince feuille d'aluminium $a a$. On peut faire le vide de Crookes dans la boîte étanche $\mathrm{ABCD}$, reliée à la trompe à mercure.

Quand on opère à la pression atmosphérique, la conductibilité de l'air sous l'influence des rayons est considérable. Lorsque le métal M de la feuille intérieure est différent du métal $\mathrm{N}$ des fenêtres $f$ et des faces internes de la boîte $\mathrm{ABCD}$, le système $(\mathrm{M} \mid \mathrm{N})$ fonctionne comme une pile dont la force électromotrice fait dévier l'électromètre. On peut, par la méthode d'opposition du quartz piézo-électrique de M. J. Curie, mesurer le courant électrique nécessaire pour maintenir l'électromètre au potentiel zéro ; ou bien on peut, sans agir sur le quartz, ramener l'électromètre à demeurer au zéro en intercalant en $\mathrm{E}$, entre la boîte $\mathrm{ABCD}$ et la terre, une force électromotrice convenable $e_{0}$ prise en dérivation sur le circuit d'un daniell.

Dans ces conditions, si l'on fait le vide dans l'appareil, l'équilibre 
de l'électromètre se maintient d'abord avec la mème force él(cetromotrice $e_{0}$ de compensation, tant que la pression ne s'est pas abaissée jusqu'à l'ordre de grandeur du millimètre (seulement, le courant qui prend naissance en l'absence de $e_{0}$ devient de plus en plus failıle). Pour des pressions inférieures, la force électromotrice de compensation est modifiée. Elle dépasse bientôt celle d'un daniell, augmente constamment et semble croître au delà de toute limite à mesure qu'on se rapproche du vide de Crookes. Si l'on rétablit en $\mathrm{E}$ la force électromotrice primitive $e_{0}$ qui compensait le phénomène à la pression atmosphérique, on peut, à l'aide dı quartz, mesurer le courant nécessaire pour maintenir l'électromètre au zéro. Ce courant, qui apparaît aux pressions de l'ordre du millimètre, augmente d'abord légèrement avec la raréfaction de l'atmosphère, puis devient sensiblement constant pour le vide de Crookes.

$\mathrm{Si}$, par exemple, le métal intérieur $\mathrm{M}$ est du platine, et si le métal $\mathrm{N}$ des parois internes de la boîte $\mathrm{ABCD}$ est de l'aluminium, il faut maintenir l'aluminium à un potentiel négatif (inférieur en valeur absolue à 1 daniell) pour obtenir la compensation à la pression atmosphérique.

Dans le vide de Crookes, cette force électromotrice n'est plus suffisante, et il faudrait porter l'aluminium à un potentiel régatif de valeur absolue égale à 30 volts environ, si l'on voulait obtenir la compensation. Si l'on maintient la force électromotrice $e_{0}$ qui compensait le phénomène à la pression atmosphérique, on constate que, dans le vide de Crookes, sous l'action des rayons de Röntgen, le platine se charge positivement. Le courant de charge, mesuré à l'aide du quartz, est de l'ordre de grandeur de $10^{-10}$ ampère quand on utilise, à travers les fenêtres $f$ recouvertes d'alıminium mince, une surlace d'environ 30 centimètres carrés placée à 6 centimètres de la source $l$ des rayons de Röntgen.

Ce courant est assez faible pour qu'on puisse dire: Tant qu'on n'opère pas dans un gaz raréfié, les rayons secondaires provoquent la conductibilité des gaz en y libérant d'égales quantités d'électricité positive et négative. Mais dans un gaz raréfié, l'on voit que l'influence des charges négatives des rayons secondaires apparaît; alors les rayons $\mathrm{X}$ déchargent les corps négatifs plus rapidement que les corps positifs, ou mème ils augmentent la clıarge des corps positifs. Il est remarquable que celte dissymétrie de la décharge, produite dans le vide par les rayons de Röntgen qui frappent un métal lourd, est de 
même sens que la dissymétrie de la décharge des conducteurs frappés par les rayons ultra-violets de Hertz et de Hallwachs.

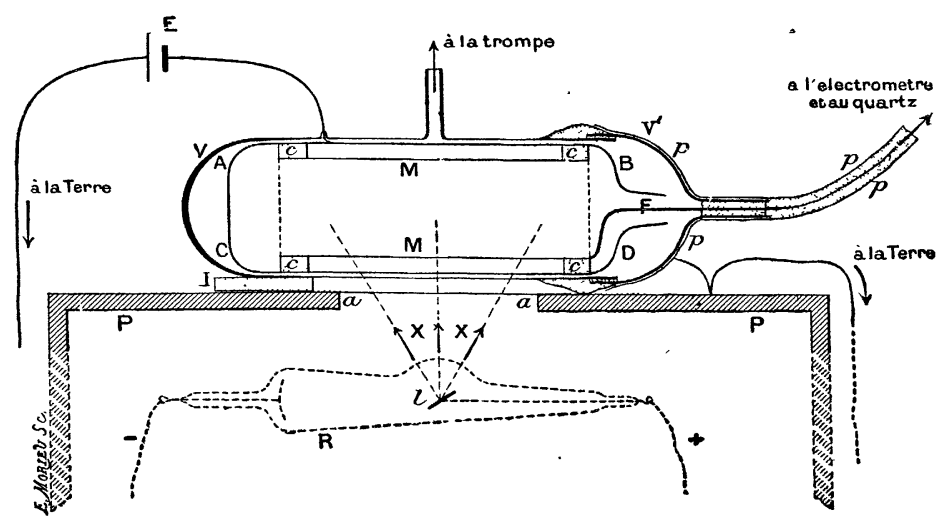

FI(i, 2.

Des résultats peu différents sont obtenus à l'aide de la disposition représentée par la fig. 2: la feuille métallique mince $\mathrm{M}$ est alors enroulée en cylindre, et la boîte plate est remplacée par un second cylindre métallique ABCD de mème axe que MM. On introduit le système MMABCD dans le récipient $\mathrm{V}$ de verre relié à la trompe à mercure, puis on ferme le récipient $\mathrm{V}$ avec le couvercle de verre $\mathrm{V}^{\prime}$ mastiqué au golaz. Avec ce second dispositif, on évite les rentrées d'air plus facilement qu'avec le premier.

Ces faits pourraient, à la rigueur, s'expliquer par une variation continue de la force électromotrice de contact, qui croîtrait dans d'énormes proportions avec le degré de vide. Cette manière de voir est peu vraisemblable (1). On explique, au contraire, nettement les phénomènes en admettant que les rayons secondaires émis par les métaux en expérience emportent avec eux de l'électricité négative et libèrent, dans le métal, la quantité complémentaire d'électricité posi-

(l) On a démontré que, si l’on fait le vide de Crookes dans un récipient renfermant un condensateur dont les armatures sont formées de deux métaux $\mathrm{M}$ et $\mathrm{N}$, la force électromotrice du couple MN n'en est pas altérée; elle est même indépendante de la nature du gaz ambiant, raréfié ou non, tant que l'on ne chauffe pas les métaux $\mathrm{M}$ et $\mathrm{N}$ dans le vide de manière à en faire dégager les gaz inclus et à les remplacer par un autre gaz (Botromley, B.-A. Report, 1883̈; Spiers, Phil. Mag., t. XLIX, particulièrement p. 70, janvier 1900.) 
tive. Le platine transformant les rayons de Röntgen considírahlement plus que l'aluminium, son émission d'électricité négative est de beaucoup plus considérable que l'émission opposée de l'aluminium, et le platine se charge positivement.

On peut renverser le phénomène en mettant l'aluminium en $M$ à l'intérieur et le platine mince $\left(\frac{1}{100}\right.$ de millimètre $)$ autour de $\mathrm{M}$, en $\operatorname{ABCD} f(f g .1)$ ou $\Lambda \mathrm{BCD}(f g .2)$. On constate alors que l'aluminium intérieur M, soumis à l'émission secondaire du platine, recueille de l'électricité négative.

Nous avons fait varier la nature des métaux et constaté en particulier que le plomb et le platine sont parmi les métaux qui émettent le plus de charges négatives sous l'action des rayons X. Viennent ensuite l'étain et le zinc. Quant à l'aluminium, des expériences faites avec une enceinte de Faraday tapissée extérieurement d'aluminium et recevant des rayons de Röntgen semblent montrer que les rayons secondaires assez pénétrants de ce corps sont, comme les rayons de Röntgen générateurs, dont ils diffèrent peu, sensiblement dépourvus de charge électrique.

L'intensité des charges électriques négatives des rayons secondaires du métal M, étudiées avec la disposition de la fig. 2, ne s'affaiblit pas considérablement lorsqu'on compare un appareil dont l'enveloppe de verre $\mathrm{V}$ est relativement mince ( 1 millimètre) à un autre où elle est plus épaisse (3 millimètres). Avec le dispositif de la fig. 1, les rayons X pénétrant dans la boîte $\mathrm{ABCD}$ par les fenêtres àtravers une feuille d'aluminium d'épaisseur de $\frac{1}{10}$ de millimètre, l'interposition d'une glace de verre de à millimètres sur le trajet des rayons de Röntgen affaiblit le phénomène, mais en le laissant comparable à ce qu'il était d'abord; l'interposition d'une lame d'aluminium d'un demimillimètre sur le trajet des rayons de Röntgen réduit à peine (de moins de $\frac{1}{100}$ ) l'électrisation négative des rayons secondaires du platine. Les charges négatives des rayons secondaires proviennent donc surtout de l'action exercée sur le métal $M$ par les rayons $X$ les - plus pénétrants du faisceau incident. Ce fait est analogue à celui qui a été signalé à propos de l'activité électrique des rayons secondaires ( ${ }^{(1)}$, mais il est ici encore bien plus marqué.

(1) G. Sagnac, De l'optique des rayons de Röntgen et des rayons secondaires qui en dérivent. Paris, Gauthier-Villars, 1900, p. 105 et 132. 
Le rapprochement précédent est en accord avec celui que l'on peut faire au sujet du pouvoir de pénétration des charges négatives lancées par le métal $M$. Quand, au lieu d'opérer dans le vide, nous avons opéré en plongeant le condensateur MN dans un diélectrique tel que la paraffine, l'ébonite, le phénomène de l'émission d'électricité négative de $\mathrm{M}$ en $\mathrm{N}$ disparaissait sensiblement. Dans le cas seulement où $\mathrm{M}$ et $\mathrm{N}$ n'étaient séparés que par une fraction de millimètre de paraffine, l'électromètre accusait encore une faible charge correspondant à des courants de l'ordre de $10^{-12}$ ampère; les variations de ces faibles courants avec la nature des métaux $\mathrm{M}$ et $\mathrm{N}$ s'accordaient à faire penser qu'ils étaient dus à l'émission par les métaux lourds de charges négatives rapidement absorbées par la paraffine au voisinage du métal.

Une expérience directe a d'ailleurs montré le faible pouvoir de transmission ( $\left.{ }^{1}\right)$ de l'émission électrique du plomb, par exemple : une moitié longitudinale du cylindre de plomb épais MM $(f g .2)$ est recouverte d'une feuille d'aluminium battu, dont l'épaisseur calculée d'après la surface, le poids et la densité 2,7 est de $\mathbf{0 , 4 6}$ micron. Les rayons $\mathrm{X}$ frappant le côté nu du cylindre, on observe, à la pression $0^{\mathrm{mm}}, 001$ de mercure, un courant de l'ordre de $10^{-10}$ ampère-dû au bombardement d'électricité négative issue du plomb nu MM; c'està-dire que le plomb MM se charge de la quantité complémentaire d'électricité positive, et il faut, pour le maintenir au potentiel zéro pendant $32^{\mathrm{s}}, 2$, disposer sur le plateau du quartz piézo-électrique une masse de $ّ 00$ grammes en l'abandonnant progressivement à l'action de son poids. L'appareil $\mathrm{VV}^{\prime}$, une fois retourné de $180^{\circ}$ autour de son axe, de manière que les rayons $X$ frappent maintenant la face de plomb recouverte d'aluminium battu, l'émission d'électricité négative par le plomb à travers cette feuille d'aluminium correspond à un

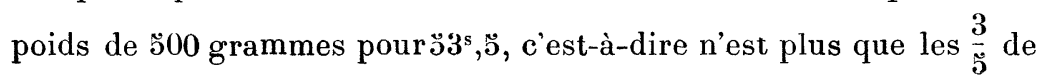
celle du plomb nu. Ce coefficient de transmission des charges électriques est assez peu différent de celui que présenteraient les rayons cathodiques extérieurs à un tube à vide dans les expériences de

(1) Latransmission étudiée ici peut avoir lieu en partie ou en totalité par diffusion postérieure; la même remarque s'applique d'ailleurs à la transmission de l'action électrique de décharge ou de l'action radiographique des rayons secon. - daires des métaux lourds, telle qu'elle a été, dans certains cas, étudiée par l'un de nous (G. Sagnic, loc. cit., p. 89 et 94 ). 


\section{CURIE ET SAGNAC. - TRANSFORMATION DES R AYONS X}

Lenard. Il est aussi comparable à'celui de l'action électrique de décharge des rayons secondaires, déjà étudié et à celui de l'action radiographique $\left({ }^{1}\right)$.

Il importe de remarquer que les mesures des courants d'électricité négative issue des métaux lourds frappés par les rayons $\mathrm{X}$ onl, pour des conditions expérimentales données, un sens absolu; la quantité d'électricité transportée par les rayons secondaires est, dans un vide suffisamment poussé, indépendante de la distance parcourue par les rayons secondaires; au contraire, on sait que les intensités des actions électriques radiographiques ou radioscopiques des rayons secondaires et aussi des rayons $\mathrm{X}$, dépendent du mode d'utilisation des rayons et, en particulier, de l'épaisseur d'air du condensateur électrique et de la couche photographique ou luminescente qui les reçoit $\left({ }^{2}\right)$. Il ne paraît d'ailleurs y avoir aucun lien simple entre l'énergie des rayons secondaires, telle qu'on pourrait la mesurer au moyen d'un bolomètre fondé sur l'échauffement d'un métal par ces rayons et la quantité d'électricité négative qu'ils transportent. L'ensemble des faits observés conduit à penser que l'émission électrique secondaire des métaux lourds possède des propriétés analogues à celles des rayons cathodiques et des rayons déviables du radium : les particules d'électricité négative des rayons sont capables de dissocier l'électricité neutre des particules des gaz en quantités d'électricités positive et négative, considérablement supérieures à la quantité d'électricité négative des rayons, tant du moins que le gaz étudié n'est pas trop raréfié. Il ne faut pas confondre la production de ces rayons cathodiques, qui, dans le vide et mème en l'absence de tout champ électrique, émanent du métal $M$ frappé par les rayons $X$, avec la production déjà signalée par l'un de nous $\left({ }^{3}\right)$ d'un flux d'électricité soit positive, soit négative, dans un gaz soumis au champ électrique.

L'action des rayons $\mathrm{X}$ et des rayons secondaires sur les gaz n'est pas essentiellement différente de l'action des rayons ultra-violets étudiés par Lenard (4). D'autre part,l'émission des rayons cathodiques par un métal lourd que frappent les rayons $\mathrm{X}$ n'est pas plus étrange que le phénomène analogue produit par les rayons ultra-violets:

(') G. SaGNaC, loc. cit., p. 9'.

(2) G. Sagnac, loc cil., p. 131.

(3) Sagnac, C. R. du 5 février 1900 et l'article précédent: Nouvelles recherches

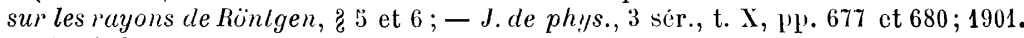

(4) Gf. loc. cit., \& 7. J. cle phys., $3^{\mathrm{e}}$ série, t. X, 1) 683. 
BOUASSE. - PETITES OSGILLATIONS DE TORSION 21 le professeur Righi (') et, plus récemment, le professeur P. Lenard $\left({ }^{2}\right)$ les professeurs E. Meritt et O.-M. Stewart $\left({ }^{3}\right)$ ont en effet trouvé qu'un métal frappé par les rayons ultra-violets émet un flux d'électricité négative, même lorsque la surface métallique frappée par les rayons n'est pas électrisée. Cette émission a les caractères de rayons cathodiques particulièrement absorbables et l'étude n'a pu en être faite par le professeur Lenard que dans le vide de Crookes.

L'électrisation négative des rayons secondaires fournit donc une analogie nouvelle entre les rayons $\mathrm{X}$ et les rayons ultra-violets. Il devient alors de plus en plus probable qu'il y a, dans les rayons secondaires, des rayons non électrisés de l'espèce même des rayons $\mathrm{X}$ incidents qui les produisent en se diffusant ou se transformant. 